\title{
Evaporation of an intensively heated sessile droplet into the open atmosphere
}

\author{
Andrey Semenov ${ }^{1,2, a}$, Geniy Kuznetsov ${ }^{3}$, Dmitry Zaitsev ${ }^{1}$ \\ ${ }^{1}$ Kutateladze Institute of Thermophysics, 630090, Lavrentiev ave. 1, Novosibirsk, Russia \\ ${ }^{2}$ Novosibirsk State University, 630090, Pirogova str. 2, Novosibirsk, Russia \\ ${ }^{3}$ National Research Tomsk Polytechnic University, 30, Lenin Ave, 634050, Tomsk, Russia
}

\begin{abstract}
Evaporation of a heated sessile water drop was studied experimentally at a temperature difference between the substrate and surrounding atmosphere from 30 to $60^{\circ} \mathrm{C}$. The studies were performed on the substrates with micro- and nanocoatings with different wettability. The features of evaporation were studied for the pinned, partially pinned and depinned contact line (solid-liquid-gas interface). It is found that during the evaporation process the specific evaporation rate (mass loss per unit of the drop surface area) increases, particularly at the last stage of the drop lifetime.
\end{abstract}

\section{Introduction}

Among the effective solutions for removing high heat flux densities, there are the systems of spray cooling [1] as well as the systems using stratified two-phase flow in a microchannel [2]. Under the certain conditions, heat in these systems is removed due to intensive evaporation of liquid droplets that are in contact with hot surface. The process of liquid drop evaporation has been actively investigated in the last decade (see [3-6] and references therein). In most experimental and theoretical studies found in the literature, the isothermal evaporation of water droplets was studied. Research on nonisothermal evaporation of liquid droplets with a sufficiently high temperature difference between the substrate and the atmosphere is practically absent.

In this paper we study the process of evaporation of relatively large liquid droplets (drop volume varies from 100 to 400 microliters) to open atmosphere. A liquid drop is heated from below with the quasi-stationary heat transfer between the solid substrate and the drop. Ultra-pure water (by Milli-Q Company) is used as the working fluid. The use of water having a relatively high boiling point as a working fluid allows us to investigate the influence of the temperature difference between the substrate and the surrounding atmosphere on the rate of evaporation and heat transfer. The temperature difference between the substrate and the surrounding atmosphere ranges from 30 to $60 \mathrm{C}$. Three types of evaporation of the liquid droplets were studied: 1) constant contact angle (moving contact line), 2) constant surface area of contact (pinned contact line) 3) mixed type (partially pinned contact line).

\footnotetext{
${ }^{a}$ Corresponding author: semenov.itp@gmail.com
} 


\section{Experimental setup and technique of measurement}

The experiments were accomplished on the experimental stand, the scheme of which is shown in Fig. 1. The test cell is an aluminum plate $50 \mathrm{~mm}$ thick, on the underside of which the heater was attached. As a heater we used Peltier element whose size is $40 \mathrm{x} 40 \mathrm{~mm}^{2}$. On the upper side of the aluminum plate we fixed a substrate, which was a disc $52 \mathrm{~mm}$ in diameter and $3.2 \mathrm{~mm}$ thick. The substrate surface temperature was measured with the thermocouple fixed at a distance of $1-2 \mathrm{~mm}$ from the contact line of a liquid droplet. The temperature under the substrate was measured by three thermocouples of K-type. Thermocouples were located radially at different distances from the center of the disc and allow to measure the temperature distribution along the radius of the substrate. We used three different substrates. Two substrates were made of a float glass plate with two different coatings: 1) the "Spin Teflon" coating, obtained by centrifugation of molten Teflon drops (with the contact angle hysteresis defined as the difference between the advancing and the receding contact angles, $122.5^{\circ}-112.7^{\circ}=9.8^{\circ}$ ), 2) the "Spray Teflon" coating, obtained by spraying of Teflon particles (contact angle hysteresis, $119.9^{\circ}-90.6^{\circ}=29.3^{\circ}$ ). The third substrate was made of stainless steel and had a developed surface structure that was obtained by bombarding the surface with calibrated aluminum oxide particles. The roughness of this surface was about 5 microns. Relative humidity in the room during the experiments was $20-30 \%$. The room temperature was about $25^{\circ} \mathrm{C}$.

Two optical techniques were used. The first one was the shadow method, which used a planeparallel light source and a camera. The spatial resolution of the system was 6 microns per pixel and shooting frequency ranged from 3 to 8 frames per second. The shadowgraphs of the liquid drops were processed by the software package Drop Shape Analysis by KRUSS company. The second optical technique consisted of a digital video camera, which was placed atop the test section and allow to visualize the process of evaporation from the top and control the symmetry of the drop. In the event that a drop lost symmetry, the experiment was repeated.

The experiment started with a pretreatment of the studied substrate, which was thoroughly clean of the working surface; then, the entire substrate was placed into a tank containing distilled water for a day. Thereafter, in order to remove residual water from the surface, the substrate was purged with a special clean compressed air intended for cleaning optical components. A predetermined volume of a liquid drop placed on the surface, heated to the desired temperature, using an electronic syringe. The optical systems were focused, and then there was periodic shooting with the step of 10 seconds until complete evaporation of a liquid drop.

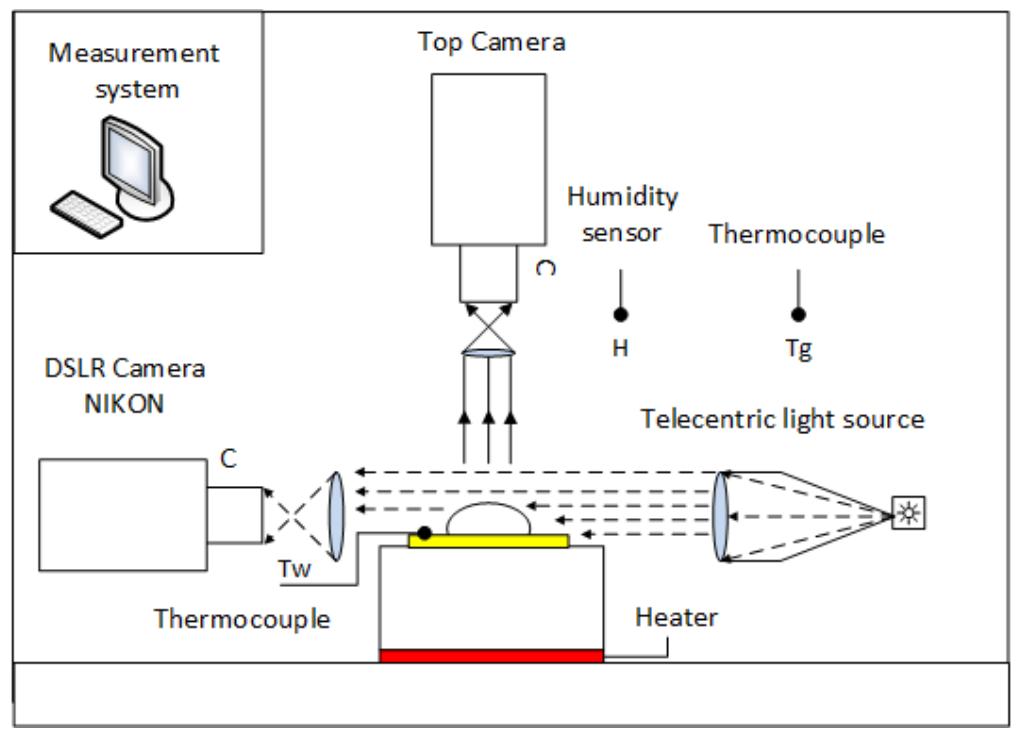

Figure 1. Schematic diagram of the experimental arrangement. 


\section{Results and discussion}

Figure 2 shows evolution of droplets during evaporation on different surfaces. The temperature of the surface for each experiment is $65^{\circ} \mathrm{C}$. In the case of the Spin Teflon the evaporation occurs with constant contact angle (moving contact line), in the case of the Stainless Steel - with constant wetted diameter (pinned contact line), in the case of the Spray Teflon - mixed type (partially pinned contact line).

\begin{tabular}{|c|c|c|c|}
\hline$t, s$ & Spin Teflon & Spray Teflon & Stainless Steel \\
\hline \multirow[t]{2}{*}{0} & $\longrightarrow$ & & \\
\hline & $V=96.65 \mu 1$ & $\mathrm{~V}=100.67 \mathrm{ul}$ & $\mathrm{V}=85.72 \mu \mathrm{l}$ \\
\hline \multirow[t]{2}{*}{200} & & & \\
\hline & $\mathrm{V}=76.47 \mu \mathrm{l}$ & $\mathrm{V}=82.77 \mu \mathrm{l}$ & $\mathrm{V}=53.88 \mu \mathrm{l}$ \\
\hline \multirow[t]{2}{*}{400} & & & \\
\hline & $\mathrm{V}=57.03 \mu \mathrm{l}$ & $\mathrm{V}=66.18 \mu \mathrm{l}$ & $\mathrm{V}=30.77 \mu \mathrm{l}$ \\
\hline \multirow[t]{2}{*}{600} & & & \\
\hline & $\mathrm{V}=44.50 \mu \mathrm{l}$ & $\mathrm{V}=49.14 \mu \mathrm{l}$ & $\mathrm{V}=10.35 \mu \mathrm{l}$ \\
\hline \multirow[t]{2}{*}{1000} & & & \\
\hline & $\mathrm{V}=17.90 \mu \mathrm{l}$ & $\mathrm{V}=22.52 \mu \mathrm{l}$ & \\
\hline
\end{tabular}

Figure 2. Evolution of droplets during evaporation on different surfaces. $\mathrm{T}_{\mathrm{w}}=65^{\circ} \mathrm{C}$.

Dependence of the specific evaporation rate of the liquid on time for different substrates is presented in Fig. 3. The rate of evaporation is calculated as the loss of the drop mass per unit drop surface area per unit time. It is seen that the specific evaporation rate increases with time (with decrease of drop volume) and at the last step of evaporation may be several times the initial value. The results are in good agreement with the findings of [7] where a comparison of the experimental and theoretical data on the dependence of the specific evaporation rate on time is presented. In contrast to [7], where the studies were carried out at a surface temperature $\mathrm{T}_{\mathrm{w}}=64^{\circ} \mathrm{C}$, in this paper we obtain new data for the surface temperatures range from 55 to $85^{\circ} \mathrm{C}$. The increase in the specific rate of evaporation is observed throughout the whole range of temperatures studied. 


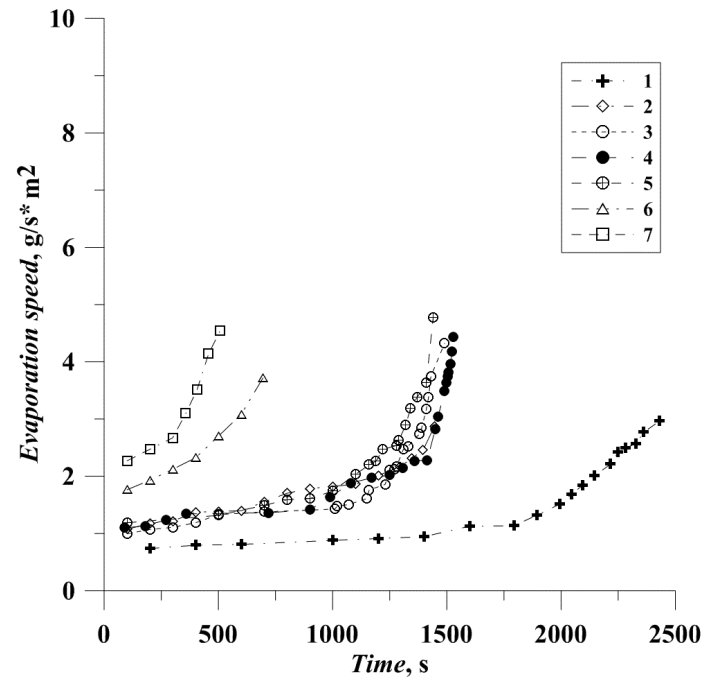

a)

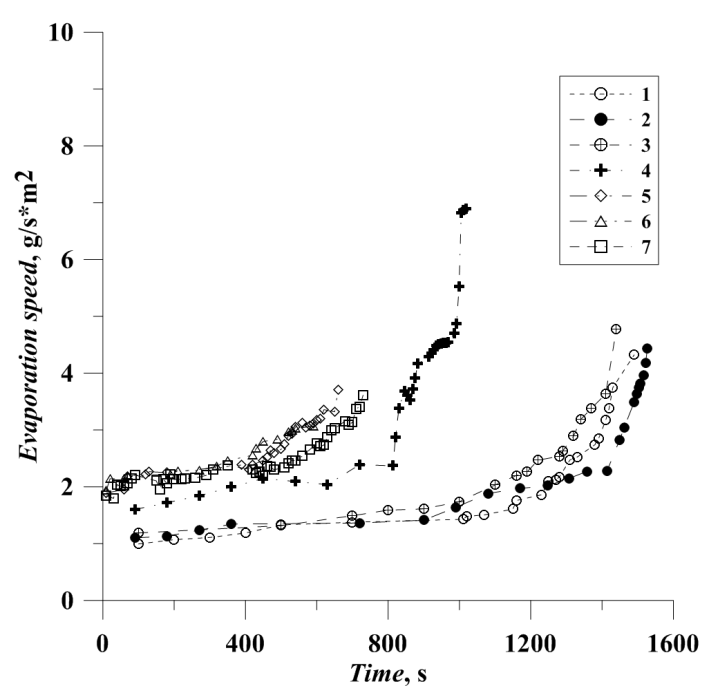

b)

Figure 3. Specific evaporation rate vs. time. a) - on the substrate with Spray Teflon coating: substrate temperature $\mathrm{T}_{\mathrm{w}}=55^{\circ} \mathrm{C}(1), 65^{\circ} \mathrm{C}(2-5)$ (series of four runs), $75^{\circ} \mathrm{C}(6), 85^{\circ} \mathrm{C}(7)$; b) - different coatings at surface temperature $\mathrm{T}_{\mathrm{w}}=65^{\circ} \mathrm{C}$ : surfaces with Spray Teflon coating (1-3), Spin Teflon coating (4), stainless steel (5-7).

\section{Acknowledgments}

Authors gratefully acknowledge support of this work by the Russian Science Foundation (Agreement No. 14-19-01755).

\section{References}

1. Kim J. International Journal of Heat and Fluid Flow, 28, 753 (2006).

2. Kabov O.A. and Zaitsev D.V. Multiphase Science and Technology, 21, 249 (2009).

3. Ajaev V.S. J. Fluid Mech., 528, 279 (2005).

4. Kuznetsov G.V., Feoktistov D.V., Orlova E.G. Journal of Engineering Physics and Thermophysics, 89, 317 (2016).

5. Kuznetsov G.V., Feoktistov D.V., Orlova E.G. Thermophysics and Aeromechanics, 23, 17 (2016).

6. Kabov O.A. and Zaitsev D.V. Doklady Physics, 58, 292 (2013).

7. Gatapova E.Ya., Semenov A.A., Zaitsev D.V., Kabov O.A., Colloids and Surfaces A: Physicochemical and Engineering Aspects, 441, 776 (2014). 